\title{
Accumbens Cholinergic Interneurons Mediate Cue-Induced Nicotine Seeking and Associated Glutamatergic Plasticity
}

\author{
Jonna M. Leyrer-Jackson, ${ }^{1}$ Michael Holter, ${ }^{2}$ Paula F. Overby, ${ }^{1}$ Jason M. Newbern, ${ }^{2}$ Michael D. Scofield, ${ }^{3}$ \\ M. Foster Olive, ${ }^{1}$ and Cassandra D. Gipson ${ }^{4}$
}

https://doi.org/10.1523/ENEURO.0276-20.2020

${ }^{1}$ Department of Psychology, Arizona State University, Tempe, AZ 85281, ${ }^{2}$ Department of Neuroscience, School of Life Sciences, Arizona State University, Tempe, AZ 85281, ${ }^{3}$ Department of Anesthesiology, Medical University of South Carolina, Charleston, SC 29425, and ${ }^{4}$ Department of Family and Community Medicine, University of Kentucky, Lexington, KY 40536

\begin{abstract}
Nicotine, the primary addictive substance in tobacco, is widely abused. Relapse to cues associated with nicotine results in increased glutamate release within nucleus accumbens core (NAcore), modifying synaptic plasticity of medium spiny neurons (MSNs), which contributes to reinstatement of nicotine seeking. However, the role of cholinergic interneurons (Chls) within the NAcore in mediating these neurobehavioral processes is unknown. Chls represent less than $1 \%$ of the accumbens neuronal population and are activated during drug seeking and reward-predicting events. Thus, we hypothesized that Chls may play a significant role in mediating glutamatergic plasticity that underlies nicotine-seeking behavior. Using chemogenetics in transgenic rats expressing Cre under the control of the choline acetyltransferase (ChAT) promoter, Chls were bidirectionally manipulated before cue-induced reinstatement. Following nicotine self-administration and extinction, Chls were activated or inhibited before a cue reinstatement session. Following reinstatement, whole-cell electrophysiology from NAcore MSNs was used to assess changes in plasticity, measured via AMPA/NMDA (A/N) ratios. Chemogenetic inhibition of Chls inhibited cued nicotine seeking and resulted in decreased $A / N$, relative to control animals, whereas activation of Chls was unaltered, demonstrating that Chl inhibition may modulate plasticity underlying cue-induced nicotine seeking. These results demonstrate that Chl neurons play an important role in mediating cue-induced nicotine reinstatement and underlying synaptic plasticity within the NAcore.
\end{abstract}

Key words: accumbens; cholinergic; glutamate; nicotine; plasticity; relapse

\section{Significance Statement}

The studies reported here are the first to address the role of cholinergic interneurons (Chls) in cue-induced nicotine seeking and in nicotine-induced changes in synaptic plasticity within nucleus accumbens medium spiny neurons (MSNs). Chemogenetic inhibition of Chls prevented cue-induced nicotine seeking and associated MSN plasticity. Additionally, these studies highlight the role of nicotinic acetylcholine receptors (nAChRs) in mediating cue-induced nicotine seeking and associated MSN morphology. Through the use of chemogenetics, behavioral assessments, and electrophysiology the results presented here highlight the importance of the cholinergic circuitry within the nucleus accumbens core (NAcore) for mediating cue-induced nicotine seeking.

Received June 24, 2020; accepted November 16, 2020; First published November 25, 2020.

The authors declare no competing financial interests.
Author contributions: J.M.L.-J., M.D.S., M.F.O., and C.D.G. designed research; J.M.L.-J., M.H., P.F.O., J.M.N., and C.D.G. performed research; M.D.S. and M.F.O. contributed unpublished reagents/analytic tools; J.M.L.-J., P.F.O., J.M.N., and C.D.G. analyzed data; J.M.L.-J. and C.D.G. wrote the paper. 


\section{Introduction}

Tobacco use disorder is the leading preventable cause of death within the United States and represents a substantial burden to public health (Prochaska and Benowitz, 2016). Self-administration of nicotine produces robust cellular adaptations within brain regions associated with drug reward, including the nucleus accumbens core (NAcore; Dani et al., 2001; Mansvelder et al., 2009; Gipson et al., 2013b; Scofield et al., 2016). The effects of nicotine on dopaminergic neurons within the ventral tegmental area (VTA) are perhaps the most prominently characterized synaptic alterations. Specifically, nicotine has been shown to increase glutamatergic input onto dopaminergic neurons, as measured by changes in AMPA/NMDA $(\mathrm{A} / \mathrm{N})$ ratios, and has been found to potentiate GABAergic input onto local VTA inhibitory neurons. Together, these findings support that nicotine exposure enhances VTA dopaminergic excitability and dopamine release into target areas, including the nucleus accumbens (Mansvelder and McGehee, 2000; Brown et al., 2010; Grieder et al., 2012). Enhanced dopaminergic release within the accumbens is hypothesized to potentiate medium spiny neuron (MSN) synapses as well as their response to glutamatergic stimulation (Pistillo et al., 2015). In support, increases in MSN spine length and density are observed in rats chronically exposed to nicotine (Brown and Kolb, 2001), an effect associated with enhanced NMDA receptor mediated currents within the NAcore. Further, drugs of abuse including nicotine induce changes in glutamatergic homeostasis within the mesocorticolimbic brain circuitry (Kalivas, 2009), thus altering glutamatergic-MSN connectivity and associated synaptic plasticity within the accumbens. In fact, cue-induced nicotine seeking has been shown to alter glutamatergic plasticity within the NAcore, enhancing the $\mathrm{A} / \mathrm{N}$ of $\mathrm{MSN}$ in a rapid and transient manner as well as morphologic increases in dendritic spine head diameter (Gipson et al., 2013b). Thus, while prior studies have explored changes in MSN synaptic plasticity because of altered dopaminergic and glutamatergic signaling, current studies have yet to explore additional mechanisms driving changes in MSN synaptic plasticity, which may underlie nicotine-seeking behaviors.

Cholinergic interneurons (Chls) account for $<1 \%$ of the cell population within the accumbens, yet they have the ability to exert powerful modulatory control over accumbens circuitry (Zhou et al., 2002; Tepper and Bolam, 2004) and may play a role in synaptic plasticity of MSNs. Chls

This work was supported by National Institutes of Health Grants DA036569, DA036569-S1, DA044479, DA046526, and DA045881 (to C.D.G.), F32AA02796201 (to J.M.L.-J.), DA042172 (to M.F.O.), and NS097537 (to J.M.N.) and by the Arizona Alzheimer's Consortium (C.D.G.).

Acknowledgements: We thank Neringa Stankeviciute, Nic Allen, Hanaa Ulangkaya, Ngoc Van Do, Jose Piña, Meghan Brickner, and Vincent Carfagno for their technical assistance.

Correspondence should be addressed to Cassandra D. Gipson at cdgips2@ uky.edu.

https://doi.org/10.1523/ENEURO.0276-20.2020

Copyright @ 2021 Leyrer-Jackson et al.

This is an open-access article distributed under the terms of the Creative Commons Attribution 4.0 International license, which permits unrestricted use, distribution and reproduction in any medium provided that the original work is properly attributed. provide most of the intrinsic cholinergic innervation of the NAcore and are widely distributed throughout the striatum (Girasole and Nelson, 2015). Additionally, Chls provide cholinergic modulation of striatal dopaminergic transmission and are known to co-release $\mathrm{ACh}$ and glutamate (Zhou et al., 2001; Cachope et al., 2012; Threlfell et al., 2012; Kljakic et al., 2017), allowing for additional modulation of MSN activity (Witten et al., 2010; Oldenburg and Ding, 2011). Importantly, NAcore Chls are involved in reward-predicting events (Atallah et al., 2014) and extracellular ACh is elevated following drug intake (Rada et al., 2001; Mansvelder et al., 2003; Crespo et al., 2006; Yee et al., 2011). In fact, acquisition of cocaine and remifentanil is paralleled with an increase in ACh release. Specifically, an overflow of ACh was observed following drug delivery, and blocking nicotinic acetylcholine receptors (nAChRs) was found to inhibit drug acquisition (Crespo et al., 2006). Together, these studies indicate that Chl-induced activation of $\mathrm{nAChRs}$ plays an important role in driving motivated drug use.

Increased glutamate release from prelimbic afferents targeting the NAcore increases cue-induced drug seeking (Stefanik et al., 2016) by modulating MSN synaptic physiology (Gipson et al., 2013b; Quintero, 2013; Alasmari et al., 2016). Given that NAcore Chls are involved in drugmotivated behavior, we hypothesized that this small population of cells mediates glutamatergic plasticity and nicotine-seeking behavior. Using choline acetyltransferase (ChAT) cre-recombinase transgenic rats (ChAT::cre), Chl activity was bidirectionally manipulated via viral administration of cre-dependent designer receptors exclusively activated by designer drugs (DREADDS) before cue-induced reinstatement to examine the modulatory role of Chls over nicotine-seeking behaviors. Whole-cell electrophysiological recordings were then conducted from NAcore MSNs to determine whether modulation of Chl activity mediates MSN synaptic plasticity measured via $\mathrm{A} /$ $\mathrm{N}$ currents.

\section{Materials and Methods}

\section{Subjects}

Sixty- to 90-d-old adult Long-Evans male $(N=17)$ and female $(N=20)$ rats were housed in a temperature and humidity-controlled animal facility on a 12/12 h dark/light reverse cycle and had ad libitum access to food and water before experimentation. All procedures were approved by the Institutional Animal Care and Use Committee (IACUC) of Arizona State University. All animals used for chemogenetic Chl manipulation were bred in-house and confirmed ChAT::cre-positive through genotyping described below. Breeder ChAT::cre-positive males (LongEvans- Tg(ChAT-cre5)5.1 Deis) were purchased from Rat Resource and Research Center (RRRC, RRC\#658) and bred in-house with Long-Evans wild-type females purchased from Envigo.

All ChAT::cre self-administration data presented here are included within a larger data set exploring the role of sex and strain on nicotine self-administration in our recently published study (Leyrer-Jackson et al., 2020). 
However, while these animals were included within the Long-Evans male and female groups, the study did not focus on the subset of animals as reported here, but rather included in a larger dataset focusing on self-administration parameters across strain and sex.

\section{ChAT::cre genotyping}

Tail snips were collected from all animals at PND 10. Subjects were genotyped using the following primers: $5^{\prime}-$ AGA GTA CAC TGT GGG CAG GA-3' (R658.F2 located within the promotor region of ChAT; forward primer) and 5'-GCA AAC GGA CAG AAG CAT TT-3' (Cre.R located in cre-recombinase reverse primer). Using standard PCRbased genotyping, all animals used were confirmed ChAT::cre transgene-positive.

\section{Surgical procedures}

All rats were anesthetized using ketamine hydrochloride $(80-100 \mathrm{mg} / \mathrm{kg}$, i.m.) and xylazine ( $8 \mathrm{mg} / \mathrm{kg}$, i.m.) and underwent surgical implantation of intravenous jugular catheters as well as stereotaxically implanted guide cannulae targeting the NAcore as previously described (Leyrer-Jackson et al., 2020; Namba et al., 2020). Intravenous jugular catheters (made from polyurethane tubing; BTPU-040; Instech) were inserted $2.5-3 \mathrm{~cm}$ into the right jugular vein and were threaded subcutaneously to the posterior side of the animal where it was connected to an indwelling back port (Instech). Dental cement (SNAP or Ortho-Jet) was used to adhere the catheter to the port. The indwelling port was sutured using 4-0 vicryl braided suture (Ethicons) subcutaneously $\sim 2 \mathrm{~cm}$ caudal from the shoulder blades. Following jugular vein catheterization, animals were immediately transferred to a rat stereotaxic frame and NAcore guide cannulae were bilaterally aimed at the NAcore according to a stereotaxic atlas (Paxinos and Franklin, 2001). Guide cannulae were bilaterally implanted $(+1.5 \mathrm{~mm}$ anterior/posterior, $\pm 2.0 \mathrm{~mm}$ medial/lateral, and $-5.5 \mathrm{~mm}$ dorsal/ventral; Paxinos and Franklin, 2001). Guide cannulae were positioned $2 \mathrm{~mm}$ dorsal to the NAcore to prevent damage to the area. Three screws were placed into the skull, where they were used as anchors (one anterior to bregma, and two posterior) for adhering dental cement to the skull to hold guide cannulae in position. Microinjectors protruded $2 \mathrm{~mm}$ past the guide cannulae into the NAcore. Immediately following implantation, viral vectors encoding DREADDs for $\mathrm{Chl}$ manipulation were infused into the NAcore of ChAT::cre animals: AAV5-hsyn-DIO-HM4D(G $\left(\mathrm{G}_{\mathrm{i}}\right)$-mCherry (inhibitory; titer: $1.2 \times 1013 \mathrm{vg} / \mathrm{ml} ; \mathrm{N}=15$; Addgene, \#44362), AAV5hsyn-DIO-rM3D $\left(\mathrm{G}_{\mathrm{s}}\right)$-mCherry (excitatory; titer: $1.3 \times 1013$ $\mathrm{vg} / \mathrm{ml} ; N=10$; Addgene, \#50485; packaged into an AAV5 vector by Penn Vector Core), or AAV5-hSyn-DIO-mCherry (control; i.e., only mCherry-expressing; titer: $1.5 \times 1013 \mathrm{vg} /$ $\mathrm{ml} ; \mathrm{N}=12$; Addgene, \#44362) at a volume of $0.5 \mu \mathrm{l}$ per hemisphere. Rats were immediately administered cefazolin $(100 \mathrm{mg} / \mathrm{kg}$, i.v.) and heparin $(10 \mathrm{U} / \mathrm{ml}$, i.v. $)$ and for seven consecutive days during the recovery period. Meloxicam ( $1 \mathrm{mg} / \mathrm{kg}$, s.c.) was given immediately and for the first $3 \mathrm{~d}$ of the recovery period. Heparin (10 U/ml, i.v.) was administered daily.

\section{Food training procedures}

All rats underwent food training on the sixth day of postoperative care. Food restriction (20 g of chow/d) was implemented a minimum of $2 \mathrm{~h}$ before food training initiation and was maintained for the duration of the experiment. Food training sessions were $15 \mathrm{~h}$ duration, and one active lever press resulted in the delivery of one food pellet [fixed-ratio-1 (FR1), schedule of reinforcement; Bio-Serv, $45 \mathrm{mg} /$ pellet) Concurrently, one food pellet was delivered every $20 \mathrm{~min}$ regardless of response. Light and tone stimuli were not paired with pellet administration. Food training criteria was set to 2:1 active to inactive lever presses throughout the session and a minimum of 200 active lever presses. All food training, self-administration, extinction, and reinstatement sessions were conducted in modular operant conditioning chambers (13 ENV-008, 15 ENV007; Med Associates), which have been previously described in detail (Overby et al., 2018; Leyrer-Jackson et al., 2020).

\section{Intravenous nicotine self-administration, extinction, and reinstatement}

Nicotine infusions $(0.02 \mathrm{mg} / \mathrm{kg} /$ infusion $)$ were paired with a compound stimulus (light+tone), and was followed by a $20-s$ timeout period. Nicotine was delivered across a 5.9-s duration, at a total volume of $0.1 \mathrm{ml}$ of nicotine was administered per infusion. Infusions were paired with cue lights, located above each lever, and a $2900-\mathrm{Hz}$ tone was presented throughout the duration of the nicotine infusion. Active lever presses during the timeout period were recorded, but did not result in additional nicotine infusions. An inactive lever was extended at all times and responses were recorded; however, presses yielded no programmed consequences or rewards. Session duration was $2 \mathrm{~h}$ in length and a FR1 schedule of reinforcement was used. During the first two sessions of self-administration, nicotine infusions were capped at 25 to prevent aversive effects that can accompany high nicotine intake. All animals were required to complete a minimum of 10 sessions before moving into extinction with the following criteria: $\geq 10$ nicotine infusions obtained and $\geq 2: 1$ active/ inactive lever press ratio. Additionally, extinction can be defined in two ways: (1) either by the lack of delivery of a reinforcer that was previously delivered after a response (e.g., responses on the active lever no longer result in nicotine infusions), or (2) as the absence of a contingency between response and reinforcer (e.g., the nicotine infusion occurs regardless of an animals' response; see Rescorla and Skucy, 1969; Lattal and Lattal, 2012). Given that the reinforcer here (the nicotine infusion) is not delivered following a time-out active lever press, the latter definition applies to these types of responses and are therefore distinct from active lever presses that directly result in the initiation of a nicotine infusion. Thus, these two types of lever presses were analyzed separately in the current study.

Following 10 non-consecutive criteria making sessions, animals were moved to the extinction phase which took place in the same boxes as self-administration. During the extinction phase, active lever presses no longer resulted 
A

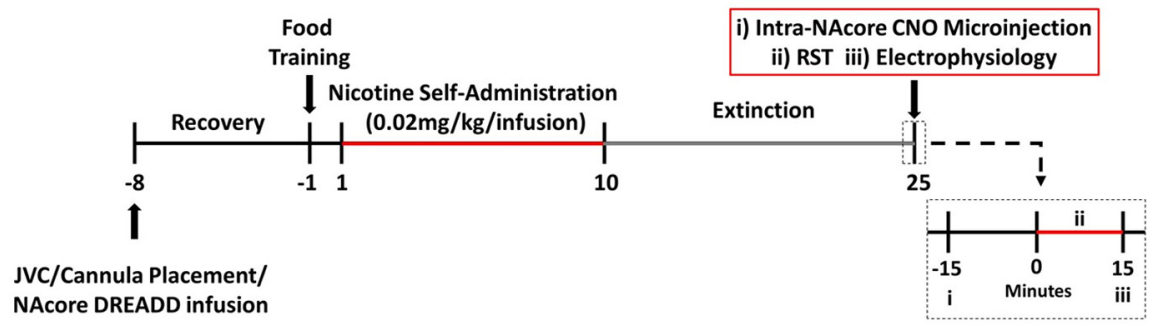

B

Self-Administration and Extinction

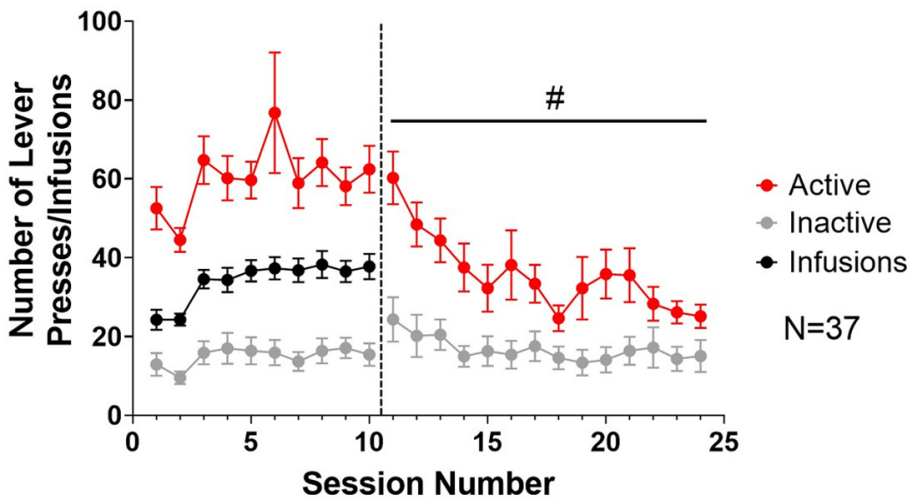

Figure 1. Nicotine self-administration and extinction. $\boldsymbol{A}$, A timeline of experimental procedures. JVC, jugular vein catheterization; RST, reinstatement. $\boldsymbol{B}$, Rats acquired nicotine self-administration, distinguishing between active (red) and inactive (gray) levers to receive intravenous infusions of nicotine (black). Active lever pressing was reduced across extinction sessions; \#p $<0.05$ represents a main effect of session on active lever pressing. The vertical dotted line in $\boldsymbol{B}$ separates self-administration sessions and extinction sessions.

in nicotine infusions or associated cues. A minimum of 14 2-h extinction sessions was required for each animal. Extinction criteria were set at $<30$ active lever presses on the last day of extinction. For reinstatement sessions, previously paired nicotine cues were presented on active lever pressing, however no nicotine infusions were delivered. Reinstatement sessions were $15 \mathrm{~min}$ in duration. Animals were then immediately killed for whole-cell electrophysiological recordings. Eight animals were removed from the current study because of not meeting self-administration criteria $(N=5)$ or lack of DREADD expression/ improper cannula placement $(N=3)$.

\section{Intra-NAcore microinjections}

For Chl manipulation, bilateral intra-NAcore microinjections of clozapine $\mathrm{N}$-oxide [CNO; $0.1 \mathrm{mg} / \mathrm{ml}$ dissolved in artificial CSF (aCSF)] at a volume of $0.5 \mu \mathrm{l} / \mathrm{hemisphere}$ were conducted 15 min before reinstatement testing (Fig. 1A). Intra-NAcore microinjections were chosen to avoid potential indirect effects of systemic CNO, since intracranial CNO administration does not exhibit DREADD-independent effects in multiple brain regions (for review, see Mahler and Aston-Jones, 2018).

\section{Electrophysiology}

Following reinstatement, animals were anesthetized with $\mathrm{CO}_{2}$ and rapidly decapitated. Brains were rapidly removed and submerged in ice-cold carbogen $\left(95 \% \mathrm{O}_{2} /\right.$ $5 \% \mathrm{CO}_{2}$ ) saturated cutting solution (cutting aCSF) containing the following: $120 \mathrm{mmol} / \mathrm{l} \mathrm{NaCl}, 25 \mathrm{mmol} / \mathrm{l}$ $\mathrm{NaHCO}_{3}, 10 \mathrm{mmol} / \mathrm{l}$ dextrose, $3.3 \mathrm{mmol} / \mathrm{KCl}, 1.23 \mathrm{mmol} /$ I $\mathrm{NaH}_{2} \mathrm{PO}_{4}, 1.8 \mathrm{mmol} / \mathrm{l} \mathrm{CaCl}_{2}$, and $2.4 \mathrm{mmol} / \mathrm{l} \mathrm{MgCl}_{2}$. Solution osmolarity was adjusted to $295 \pm 5 \mathrm{mOsm}$ and $\mathrm{pH}$ adjusted to $7.40 \pm 0.03$. Brains were then transferred to a cutting chamber of a vibrating tissue slicer (Leica, VT1000S) and 300- $\mu \mathrm{m}$-thick coronal slices of the NAcore were prepared in ice-cold cutting aCSF. Slices were then placed in a holding chamber filled with recording aCSF solution containing the following: $120 \mathrm{mmol} / \mathrm{I} \mathrm{NaCl}, 25$ $\mathrm{mmol} / \mathrm{l} \mathrm{NaHCO}, 3.3 \mathrm{mmol} / \mathrm{l} \mathrm{KCl}, 1.23 \mathrm{mmol} / \mathrm{l} \mathrm{NaH} \mathrm{PO}_{4}$, $0.9 \mathrm{mmol} / \mathrm{I} \mathrm{CaCl}_{2}, 2.0 \mathrm{mmol} / \mathrm{l} \mathrm{MgCl}_{2}$, and $10 \mathrm{mmol} / \mathrm{l} \mathrm{dex}-$ trose, osmolarity adjusted to $295 \pm 5 \mathrm{mOsm}$ and $\mathrm{pH}$ adjusted to $7.40 \pm 0.03$. During brain slicing, cannulae placement was confirmed based on cannula track marks targeting the NAcore. In the holding chamber, aCSF was continuously bubbled with carbogen $\left(95 \% \mathrm{O}_{2} / 5 \% \quad \mathrm{CO}_{2}\right)$ and incubated at $34^{\circ} \mathrm{C}$ for $45 \mathrm{~min}$ and then allowed to cool to room temperature before slice recording. Before experiments, slices were transferred to a recording chamber where they were perfused continuously at a flow rate of 1-2 $\mathrm{ml} / \mathrm{min}$ with filtered, carbogen-saturated recording aCSF solution. MSNs were visually identified using infrared DIC microscopy with an Olympus BX51WI microscope. Additionally, a green collimated LED (ThorLabs) was used to visualize mCherry-DREADD expression 
within the NAcore to ensure proper expression and location before recordings. Whole-cell recordings were made from the soma of MSNs neurons after establishing a gigaohm seal (resistance range: $1-10 \mathrm{G} \Omega$ ). Recording pipettes (7-15 $\mathrm{m} \Omega$ ), made from thin-walled capillary tubes were filled with an intracellular solution containing the following: $135 \mathrm{mmol} / \mathrm{l} \mathrm{K}$-gluconate, $12 \mathrm{mmol} / \mathrm{l} \mathrm{NaCl}, 1 \mathrm{mmol} / \mathrm{l} \mathrm{K}$ EGTA, $10 \mathrm{mmol} / \mathrm{l} \mathrm{HEPES}, 2 \mathrm{mmol} / \mathrm{l} \mathrm{Mg}$-ATP, and 0.38 $\mathrm{mmol} / \mathrm{l}$ tris-GTP. Osmolarity was adjusted to $285 \pm 5$ $\mathrm{mOsm}$ and $\mathrm{pH}$ adjusted to $7.30 \pm 0.01$. Upon establishing a giga-seal, the cell membrane was ruptured and held at $80 \mathrm{mV}$. Resting membrane potential, cellular capacitance, membrane resistance and pipet resistance were monitored throughout the duration of the recording. Only cells that exhibited thin, over shooting action potentials, normal resting membrane potential, and changes in uncompensated access resistance $<20 \mathrm{~m} \Omega$ were included in analysis. Recordings were initiated $10 \mathrm{~min}$ after cell membrane rupture to allow for diffusion of the internal solution into the cell. A stimulating electrode was placed in the dorsal region of the NAcore to active prelimbic excitatory fibers targeting the NAcore. AMPA currents, evoked by electrical stimulation, were first measured at $-80 \mathrm{mV}$. The membrane potential was then gradually increased to $+40 \mathrm{mV}$. The cell was left to stabilize at $+40 \mathrm{mV}$ for $5 \mathrm{~min}$. EPSCs composed of both AMPA and NMDA receptor mediated currents were then elicited at $+40 \mathrm{mV}$. DNQX $(20 \mu \mathrm{m})$ was then bath applied for $5 \mathrm{~min}$ and NMDA receptor mediated currents were obtained. AMPA currents were then obtained by subtracting the isolated NMDA receptor-mediated current from the whole EPSC. A/N ratios were calculated by measuring the peak amplitude of each current and taking a ratio. For DREADD function validation, see procedures outlined in (Tomek et al., 2020). All recordings were conducted using the recording software Axograph. Responses were digitized at $10 \mathrm{kHz}$ and saved on a disk using digidata interface (Molecular Devices) and analyzed offline using Axograph.

\section{Immunohistochemistry}

Slices from electrophysiology were postfixed in $4 \%$ paraformaldehyde (PFA) solution for a minimum of $2 \mathrm{~d}$. Slices were washed three times for 10 min each with PBS and placed into blocking solution containing PBST (PBS containing $0.1 \%$ Triton $\mathrm{X}-100$ ) and $5 \%$ normal donkey serum. Slices were blocked for $2 \mathrm{~h}$ at room temperature. The mouse monoclonal Anti-ChAT antibody (1:1000; Atlas Antibodies; AMAB91130) was added to the blocking solution at a concentration of 1:1000. Tissue was incubated overnight at $4^{\circ} \mathrm{C}$ with gentle rocking. Slices were washed three times for $10 \mathrm{~min}$ each using PBST. Following the third wash, slices were incubated with Alexa Fluor 488-conjugated donkey anti-mouse IgG secondary antibody (1:200; Abcam; ab205718) in PBST $(1: 200)$ for $2 \mathrm{~h}$. Slices were washed for three times using PBST under gentle agitation. Brains were mounted onto microscope slides using ProLong Gold Antifade mounting medium (ThermoFisher Scientific) and covered with cover glasses, and sealed with clear nail polish. Images were collected on a Zeiss LSM800 confocal microscope from three different tissue sections per rat with a minimum of three biological replicates. Optical sections were taken from a range of $5-15 \mu \mathrm{m}$ from the surface of the brain tissue. All images were acquired using the same acquisition parameters, including laser power, gain, and offset.

\section{Drugs and viral vectors}

(-)Nicotine tartrate (MP Biomedicals) was dissolved in $0.9 \%$ sterile saline and adjusted to $\mathrm{pH} 7.2-7.4$ with $1 \mathrm{~m}$ $\mathrm{NaOH}$. The final stock concentration was $0.2 \mathrm{mg} / \mathrm{ml}$ free base, which was adjusted for body weight to achieve an infusion concentration of $0.02 \mathrm{mg} / \mathrm{kg} / \mathrm{ml}$. CNO was purchased from Sigma-Aldrich and diluted in ACSF purchased from Tocris Biotech to $0.1 \mathrm{mg} / \mathrm{ml}$. Heparin, xylazine $(100 \mathrm{mg} / \mathrm{ml})$, cefazolin and meloxicam used at $10 \mathrm{U} / \mathrm{ml}, 8 \mathrm{mg} / \mathrm{kg} / \mathrm{ml}, 100 \mathrm{mg} / \mathrm{kg} / \mathrm{ml}$, and $1 \mathrm{mg} / \mathrm{kg}$ in $0.9 \%$ sterile saline, respectively.

\section{Data analysis}

Analysis of self-administration, extinction and reinstatement data were performed using two-way, mixed measures ANOVA with DREADD virus as a main factor and session (extinction vs reinstatement, where applicable) as a repeated-measure factor. Electrophysiological data were analyzed using a one-way ANOVA, where DREADD virus was considered a factor. Bonferroni-corrected $t$ tests post hoc, were conducted where appropriate. The effects of sex were examined in a separate oneway ANOVA to ensure no differences between groups before collapsing for analyses. Analysis of behavioral data only included animals that met self-administration criteria, had proper cannula placement, and expressed DREADDs within the NAcore. Linear regression analyses were used to explore the relationship between $A / N$ and number of active lever presses. Statistical tests were performed in GraphPad Prism 8.0, and $p<0.05$ was considered statistically significant. Values presented are represented as mean \pm SEM. All results and statistical tests ran are listed within Table 1.

\section{Results}

\section{Nicotine self-administration and extinction}

A two-way ANOVA revealed that active lever pressing remained higher than inactive lever pressing across selfadministration sessions $\left(F_{(9,780)}=349.3 ; p<0.05\right.$; Fig. $1 B)$. No interaction between session and lever was observed $(p>0.05$; Fig. $1 B$ ). We next compared reinforced and non-reinforced active lever pressing. A two-way ANOVA where session and group (reinforced and nonreinforced active lever pressing) were considered main effects revealed an effect of group $\left(F_{(1,740)}=13.6\right.$; $p<0.05)$ but not session $(p>0.05)$ and no interaction was observed $(p<0.05)$. However, a Bonferroni's post hoc comparison showed no differences between reinforced and non-reinforced lever pressing across session $(p>0.05)$. For extinction, a two-way ANOVA revealed that active lever pressing decreased across sessions $\left(F_{(13,1092)}=3.2 ; p<0.05 ;\right.$ Fig. $\left.1 B\right)$. Further, a post hoc 
Table 1: Statistical analyses in Figures 1-6

\begin{tabular}{|c|c|c|c|c|c|c|}
\hline Figure location & Behavioral test & Statistical test used & Variables/comparisons & Degree of freedom & Test value & $p$ value \\
\hline \multirow[t]{20}{*}{$1 B$} & \multirow[t]{3}{*}{ Self-administration } & \multirow[t]{2}{*}{ Two-way ANOVA } & Session & 780 & 1.8 & 0.07 \\
\hline & & & Lever & 780 & 349.3 & $<0.0001$ \\
\hline & & Interaction & 780 & 0.8 & 0.60 & \\
\hline & \multirow[t]{17}{*}{ Extinction } & \multirow[t]{2}{*}{ Two-way ANOVA } & Session & 1092 & 3.2 & $<0.001$ \\
\hline & & & Lever & 1092 & 103.7 & 0.0001 \\
\hline & & Interaction & 1092 & 1.1 & 0.33 & \\
\hline & & Bonferroni's post hoc & Active vs inactive lever: session 1 & 1092 & 5.1 & $<0.0001$ \\
\hline & & Bonferroni's post hoc & Active vs inactive lever: session 2 & 1092 & 4.0 & 0.0009 \\
\hline & & Bonferroni's post hoc & Active vs inactive lever: session 3 & 1092 & 3.4 & 0.0096 \\
\hline & & Bonferroni's post hoc & Active vs inactive lever: session 4 & 1092 & 3.2 & 0.019 \\
\hline & & Bonferroni's post hoc & Active vs inactive lever: session 5 & 1092 & 2.7 & 0.28 \\
\hline & & Bonferroni's post hoc & Active vs inactive lever: session 6 & 1092 & 3.2 & 0.02 \\
\hline & & Bonferroni's post hoc & Active vs inactive lever: session 7 & 1092 & 2.4 & 0.29 \\
\hline & & Bonferroni's post hoc & Active vs inactive lever: session 8 & 1092 & 1.4 & 0.90 \\
\hline & & Bonferroni's post hoc & Active vs inactive lever: session 9 & 1092 & 2.7 & 0.10 \\
\hline & & Bonferroni's post hoc & Active vs inactive lever: session 10 & 1092 & 3.1 & 0.03 \\
\hline & & Bonferroni's post hoc & Active vs inactive lever: session 10 & 1092 & 2.7 & 0.09 \\
\hline & & Bonferroni's post hoc & Active vs inactive lever: session 10 & 1092 & 1.6 & 0.82 \\
\hline & & Bonferroni's post hoc & Active vs inactive lever: session 10 & 1092 & 1.7 & 0.75 \\
\hline & & Bonferroni's post hoc & Active vs inactive lever: session 10 & 1092 & 1.4 & 0.90 \\
\hline \multirow[t]{3}{*}{ None } & \multirow{3}{*}{$\begin{array}{l}\text { Self-administration (reinforced vs non- } \\
\text { reinforced) }\end{array}$} & \multirow[t]{3}{*}{ Two-way ANOVA } & Session & 740 & 1.74 & 0.08 \\
\hline & & & Group (reinforced, non-reinforced) & 740 & 13.6 & 0.0002 \\
\hline & & & Interaction & 740 & 1.16 & 0.32 \\
\hline None & $\begin{array}{l}\text { Self-administration (reinforced vs non- } \\
\text { reinforced) }\end{array}$ & Bonferroni's post hoc & Reinforced vs non-reinforced, all sessions & 740 & $0.44-2.22$ & 0.27 to $>0.999$ \\
\hline \multirow[t]{3}{*}{$2 A$} & \multirow{3}{*}{$\begin{array}{l}\text { Self-administration: sex differences, active } \\
\text { lever pressing }\end{array}$} & \multirow[t]{3}{*}{ Two-way ANOVA } & Sex (male, female) & 360 & 1.64 & 0.20 \\
\hline & & & Session & 360 & 1.30 & 0.24 \\
\hline & & & Interaction & 360 & 0.33 & 0.97 \\
\hline \multirow[t]{3}{*}{$2 B$} & \multirow{3}{*}{$\begin{array}{l}\text { Self-administration: sex differences, inactive } \\
\text { lever pressing }\end{array}$} & \multirow[t]{3}{*}{ Two-way ANOVA } & Sex (male, female) & 360 & 0.63 & 0.77 \\
\hline & & & Session & 360 & 8.57 & 0.004 \\
\hline & & & Interaction & 360 & 0.21 & 0.99 \\
\hline $2 B$ & $\begin{array}{l}\text { Self-administration: sex differences, inactive } \\
\text { lever pressing }\end{array}$ & Bonferroni's post hoc & Male vs female all sessions & 360 & $0.37-1.55$ & $>0.999$ \\
\hline \multirow[t]{3}{*}{$2 \mathrm{C}$} & \multirow[t]{3}{*}{ Self-administration: sex differences, infusions } & \multirow[t]{3}{*}{ Two-way ANOVA } & Sex (male, female) & 360 & 0.34 & 0.56 \\
\hline & & & Session & 360 & 3.47 & 0.004 \\
\hline & & & Interaction & 360 & 0.31 & 0.97 \\
\hline $2 C$ & Self-administration: sex differences, infusions & Bonferroni's post hoc & Male vs female: all sessions & 360 & $0.03-1.08$ & $>0.999$ \\
\hline $2 D$ & $\begin{array}{l}\text { Self-administration: sex differences, total num- } \\
\text { ber of infusions }\end{array}$ & Unpaired Student's $t$ test & Sex (male, female) & 36 & 0.25 & 0.80 \\
\hline \multirow[t]{3}{*}{$2 E$} & Extinction: sex differences, active lever & Two-way ANOVA & Sex (male, female) & 504 & 2.55 & 0.11 \\
\hline & pressing & & Session & 504 & 2.28 & 0.006 \\
\hline & & & Interaction & 504 & 0.75 & 0.71 \\
\hline $2 E$ & $\begin{array}{l}\text { Extinction: sex differences, active lever } \\
\text { pressing }\end{array}$ & Bonferroni's post hoc & Male vs female: all sessions & 504 & $0.02-1.67$ & $>0.999$ \\
\hline $2 F$ & Extinction: sex differences, inactive lever & Two-way ANOVA & Sex (male, female) & 504 & 5.46 & 0.02 \\
\hline & pressing & & Session & 504 & 0.70 & 0.76 \\
\hline & & & Interaction & 504 & 0.48 & 0.93 \\
\hline $2 F$ & $\begin{array}{l}\text { Extinction: sex differences, inactive lever } \\
\text { pressing }\end{array}$ & Bonferroni's post hoc & Male vs female: all sessions & 504 & $0.0007-1.7$ & 0.74 to $>0.999$ \\
\hline $3 A$ & Self-administration: DREADD treatment, active & Two-way ANOVA & Treatment $\left(\mathrm{G}_{\mathrm{s}}, \mathrm{G}_{\mathrm{i}}\right.$, control) & 350 & 8.72 & 0.0002 \\
\hline & lever pressing & & Session & 350 & 1.21 & 0.29 \\
\hline & & & Interaction & 350 & 0.56 & 0.92 \\
\hline $3 B$ & Self-administration: DREADD treatment, inac- & Two-way ANOVA & Treatment $\left(\mathrm{G}_{\mathrm{s}}, \mathrm{G}_{\mathrm{i}}\right.$, control) & 350 & 6.65 & 0.002 \\
\hline & tive lever pressing & & Session & 350 & 1.04 & 0.411 \\
\hline & & & Interaction & 350 & 0.65 & 0.86 \\
\hline $3 C$ & Self-administration: DREADD treatment, & Two-way ANOVA & Treatment $\left(\mathrm{G}_{\mathrm{s}}, \mathrm{G}_{\mathrm{i}}\right.$, control) & 350 & 0.57 & 0.56 \\
\hline & infusions & & Session & 350 & 3.18 & 0.001 \\
\hline & & & Interaction & 350 & 0.33 & 0.99 \\
\hline $3 D$ & $\begin{array}{l}\text { Self-administration: DREADD treatment, total } \\
\text { number of infusions }\end{array}$ & One-way ANOVA & Treatment $\left(\mathrm{G}_{\mathrm{s}}, \mathrm{G}_{\mathrm{i}}\right.$, control) & 35 & 1.25 & 0.90 \\
\hline & & (Co & inued) & & & \\
\hline
\end{tabular}


Table 1: Continued

\begin{tabular}{|c|c|c|c|c|c|c|}
\hline Figure location & Behavioral test & Statistical test used & Variables/comparisons & Degree of freedom & Test value & $p$ value \\
\hline \multirow[t]{3}{*}{$3 E$} & Extinction: DREADD treatment, active lever & Two-way ANOVA & Treatment $\left(\mathrm{G}_{\mathrm{s}}, \mathrm{G}_{\mathrm{i}}\right.$, control) & 504 & 12.74 & $<0.0001$ \\
\hline & pressing & & Session & 504 & 2.21 & 0.008 \\
\hline & & & Interaction & 504 & 0.80 & 0.75 \\
\hline \multirow[t]{3}{*}{$3 F$} & Extinction: DREADD treatment, inactive lever & Two-way ANOVA & Treatment $\left(\mathrm{G}_{\mathrm{s}}, \mathrm{G}_{\mathrm{i}}\right.$, control) & 504 & 10.52 & $<0.0001$ \\
\hline & pressing & & Session & 504 & 0.67 & 0.79 \\
\hline & & & Interaction & 504 & 0.21 & $>0.99$ \\
\hline \multirow[t]{3}{*}{$4 C$} & Reinstatement & Two-way ANOVA & Treatment $\left(\mathrm{G}_{\mathrm{s}}, \mathrm{G}_{\mathrm{i}}\right.$, control) & 70 & 8.43 & 0.0005 \\
\hline & & & Session & 70 & 13.24 & 0.0005 \\
\hline & & & Interaction & 70 & 6.84 & 0.0019 \\
\hline $4 C$ & & Bonferroni's post hoc & Control extinction vs control reinstatement & 70 & 4.43 & 0.0005 \\
\hline $4 C$ & & Bonferroni's post hoc & $\mathrm{G}_{\mathrm{s}}$ extinction vs $\mathrm{G}_{\mathrm{s}}$ reinstatement & 70 & 2.2 & 0.03 \\
\hline $4 C$ & & Bonferroni's post hoc & $\mathrm{G}_{\mathrm{i}}$ extinction vs $\mathrm{G}_{\mathrm{i}}$ reinstatement & 70 & 0.55 & 0.999 \\
\hline $4 D$ & $\mathrm{~A} / \mathrm{N}$ ratio & Ordinary one-way ANOVA & Treatment $\left(\mathrm{G}_{\mathrm{s}}, \mathrm{G}_{\mathrm{i}}\right.$, control) & 25 & 5.7 & 0.009 \\
\hline $4 D$ & & Bonferroni's post hoc & Control vs inhibitory & 25 & 3.3 & 0.009 \\
\hline $5 A$ & Membrane capacitance & Ordinary one-way ANOVA & Treatment $\left(\mathrm{G}_{\mathrm{s}}, \mathrm{G}_{\mathrm{i}}\right.$, control) & 25 & 0.38 & 0.69 \\
\hline $5 B$ & Resting membrane potential & Ordinary one-way ANOVA & Treatment $\left(\mathrm{G}_{\mathrm{s}}, \mathrm{G}_{\mathrm{i}}\right.$, control) & 25 & 3.3 & 0.054 \\
\hline $5 C$ & NMDA decay & Ordinary one-way ANOVA & Treatment $\left(\mathrm{G}_{\mathrm{s}}, \mathrm{G}_{\mathrm{i}}\right.$, control) & 25 & 5.7 & 0.009 \\
\hline 6 & $\begin{array}{l}\text { Reinstatement active lever pressing correlation } \\
\text { to } A / N\end{array}$ & Linear regression analysis & Active lever pressing vs $\mathrm{A} / \mathrm{N}$ ratio & 26 & 12.37 & 0.002 \\
\hline
\end{tabular}

Bolded values indicate statistical significance.

comparison revealed that at the beginning of extinction, active lever pressing was higher than inactive lever pressing $\left(t_{(1092)}=5.1 ; p<0.05\right.$; Fig. $\left.1 B\right)$. However, for the last four sessions, active and inactive lever pressing were not statistically different $(p>0.05$; Fig. $1 B)$.

\section{Sex differences in self-administration and extinction}

We next examined differences between males and females throughout self-administration and extinction using a two-way ANOVA, where session and sex (male and female) were considered factors. No differences in active lever pressing between sexes across self-administration sessions were observed ( $p>0.05$; Fig. 2). An effect of session on inactive lever pressing $\left(F_{(1,360)}=8.6 ; p<0.01\right)$ and infusions $\left(F_{(1,360)}=3.5 ; p<0.01\right)$ was observed. However, post hoc analysis revealed no within session differences between males and females for inactive lever pressing $(p>0.05)$ or infusions $(p>0.05)$, suggesting that females and males do not differ within sessions.

During extinction, an effect of session on active lever pressing was observed throughout sessions $\left(F_{(13,504)}=2.28\right.$; $p<0.01)$. A post hoc comparison revealed no differences between sexes within sessions $(p>0.05)$. Additionally, an effect of sex on inactive lever pressing was observed throughout extinction sessions $\left(F_{(13,504)}=5.46 ; p<0.05\right)$, yet a post hoc comparison revealed no differences within sessions. Additionally, no differences in the total number of infusions earned across the 10-criteria making sessions were observed between male $(N=17)$ and female $(N=20)$ animals $(p>0.05$; Fig. 2). These results are consistent with our previous study, where we found no differences in total infusions or number of infusions across self-administration sessions between Long-Evans male and female rats (Leyrer-Jackson et al., 2020).

\section{$D R E A D D$-type differences in self-administration and} extinction

A two-way ANOVA where session and treatment (DREADD type; control: $N=12$; excitatory: $N=10$ and inhibitory: $N=15$ DREADD-expressing animals) were considered factors revealed a significant effect of treatment $\left(F_{(2,350)}=8.7 ; p<0.001\right)$ but not session $\left(F_{(9,350)}=\right.$ $1.2 ; p>0.05)$ on active lever pressing. However, no interaction was observed $(p>0.05)$. . A significant effect of treatment $\left(F_{(2,350)}=6.7 ; p<0.01\right)$ but not session $\left(F_{(9,350)}=1.0 ; p>0.05\right)$ on inactive lever pressing. However, no interaction was observed $(p>0.05)$. A post hoc comparison revealed no differences between DREADD type within sessions $(p>0.05)$. A two-way ANOVA also revealed a significant effect of session $\left(F_{(9,350)}=3.2 ; p<0.01\right)$ but not DREADD type $\left(F_{(2,350)}=\right.$ $0.6 ; p>0.05)$ on nicotine infusions throughout self-administration. No interaction was observed. Additionally, a post hoc comparison revealed no differences between DREADD type within sessions. Lastly, control, excitatory, and inhibitory DREADD-expressing animals did not differ in total number of nicotine infusions earned across the 10-criteria making sessions ( $p>0.05$; Fig. 3$)$. An effect of treatment and session on active lever pressing was observed throughout extinction sessions $\left(F_{(2,504)}=12.75 ; p<0.01\right.$ and $F_{(13,504)}=2.2 ; p<0.01$, respectively). No interaction was observed $(p>0.05)$. Additionally, an effect of treatment on inactive lever pressing was observed throughout extinction sessions $\left(F_{(2,504)}=10.5 ; p<0.05\right)$, yet a post hoc comparison revealed no differences between DREADD groups within sessions.

\section{ChI DREADD validation using immunohistochemistry and electrophysiology}

Using immunohistochemistry, all three vectors (two credependent DREADDs and the cre-dependent mCherry-expressing control) were validated to ensure specific targeting of Chls. DREADD-labeled neurons (mCherry; Fig. 4A, left panel) co-expressed ChAT protein (Fig. $4 A$, middle and right panel). Recordings from mCherry-tagged DREADD-labeled Chls confirmed functionality of all DREADDs used. Bath 
A

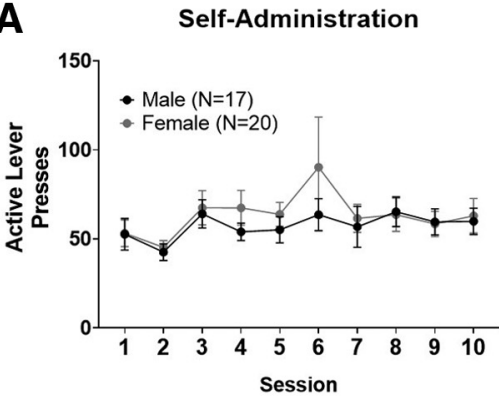

D

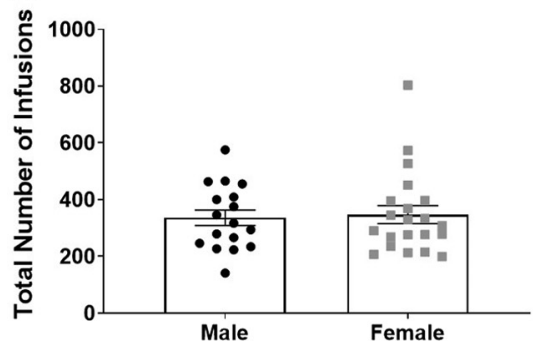

B

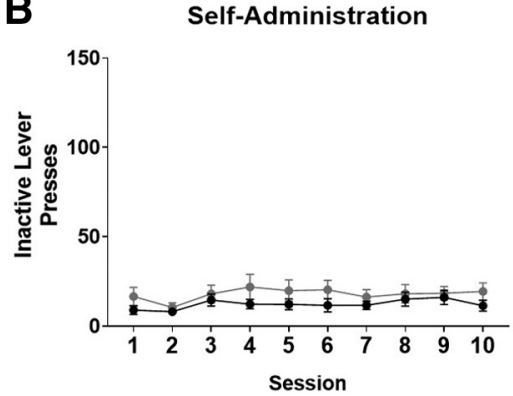

E

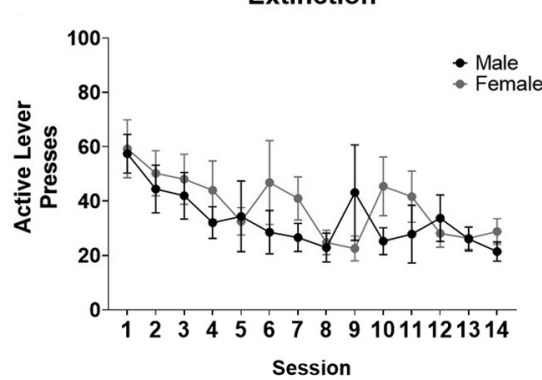

C

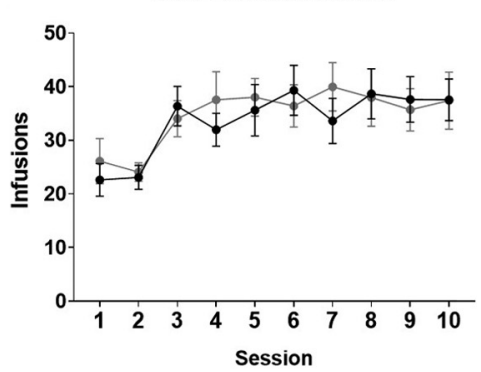

$\mathbf{F}$

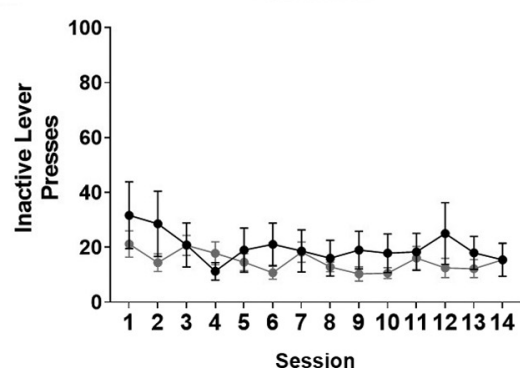

Figure 2. Male and female rats did not differ in nicotine self-administration or extinction of lever pressing. No differences were found between male and female rats in active $(\boldsymbol{A})$ or inactive $(\boldsymbol{B})$ lever pressing throughout self-administration. Additionally, they did not differ in the number of infusions across self-administration sessions $(\boldsymbol{C})$ or the total number of nicotine infusions earned throughout the 10 non-consecutive criteria-making sessions $(\boldsymbol{D})$. Data points within $\boldsymbol{D}$ represent individual animal values. Males and females did not differ in active $(\boldsymbol{E})$ or inactive $(\boldsymbol{F})$ lever pressing throughout extinction. Male and female rats are depicted in black and gray, respectively. Numbers in legend of panel $\boldsymbol{A}$ represent the number of animals in each group.

A
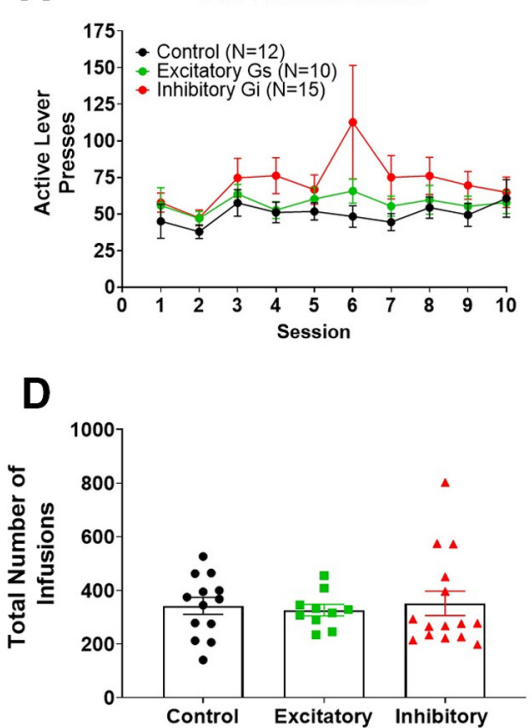

B

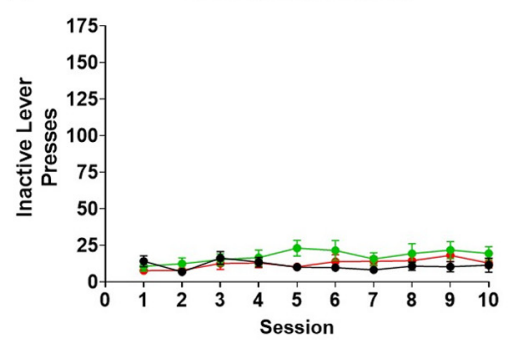

E

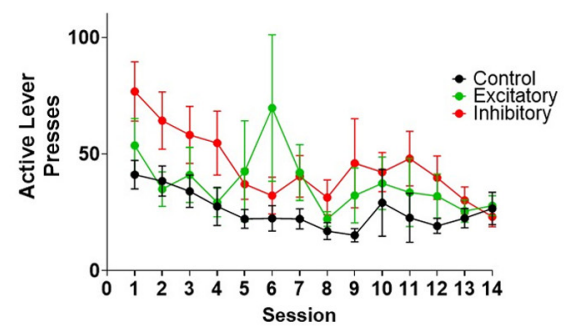

C

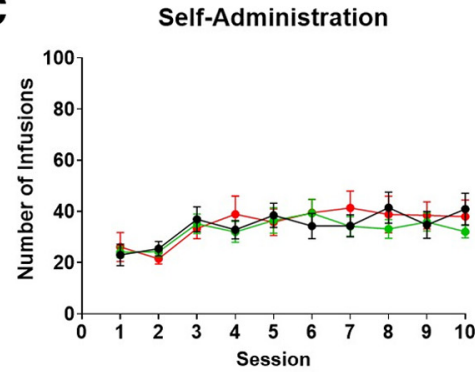

$\mathbf{F}$

Extinction

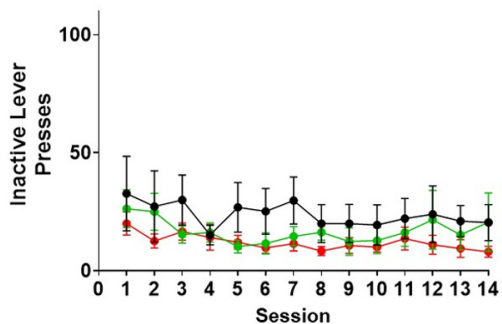

Figure 3. Control, excitatory, and inhibitory DREADD-expressing animals did not differ in rates of nicotine self-administration or extinction lever pressing. Regardless of the type of DREADD expression, animals showed no difference in active $(\boldsymbol{A})$ or inactive $(\boldsymbol{B})$ lever pressing throughout self-administration. Additionally, animals not differ in the number of infusions across self-administration sessions $(\boldsymbol{C})$ or the total number of nicotine infusions earned throughout the 10 non-consecutive criteria-making sessions $(\boldsymbol{D})$. Data points within $\boldsymbol{D}$ represent individual animal values. DREADD groups did not differ in active $(\boldsymbol{E})$ or inactive $(\boldsymbol{F})$ lever pressing throughout extinction. Control, excitatory and inhibitory DREADD-expressing rats are depicted in black, green, and red, respectively. Numbers in legend of panel $\boldsymbol{A}$ represent the number of animals in each group. 
A
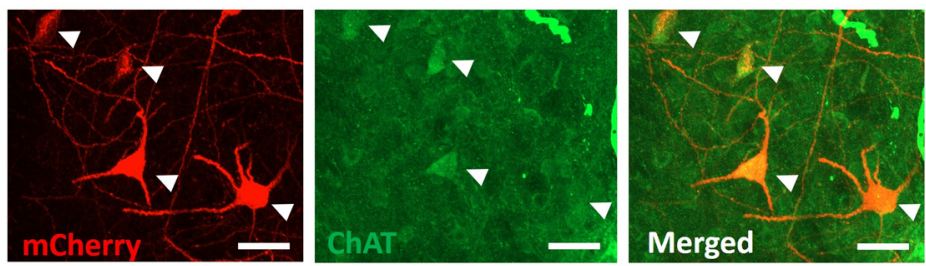

B
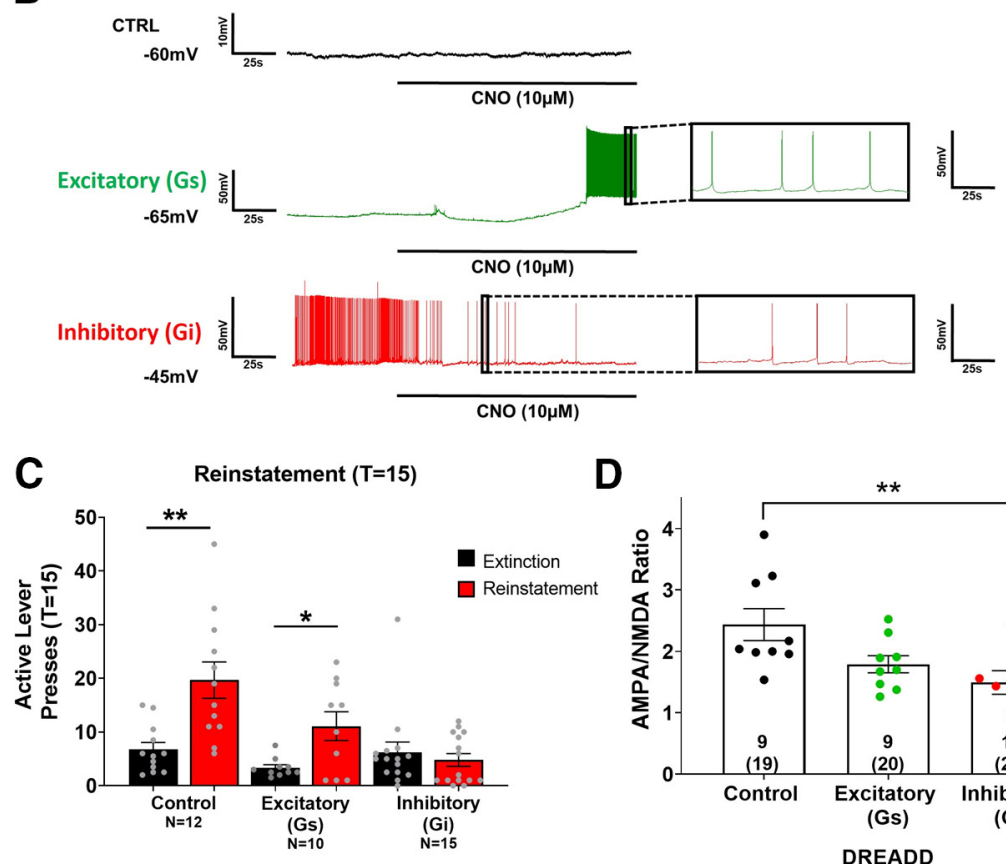

D

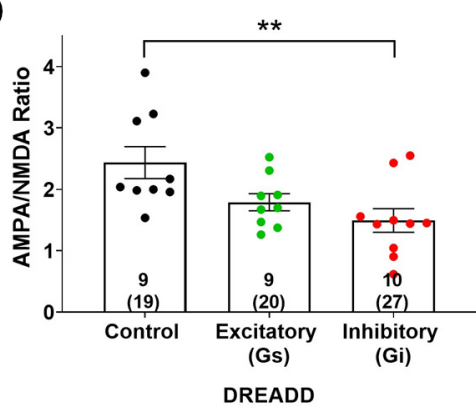

E
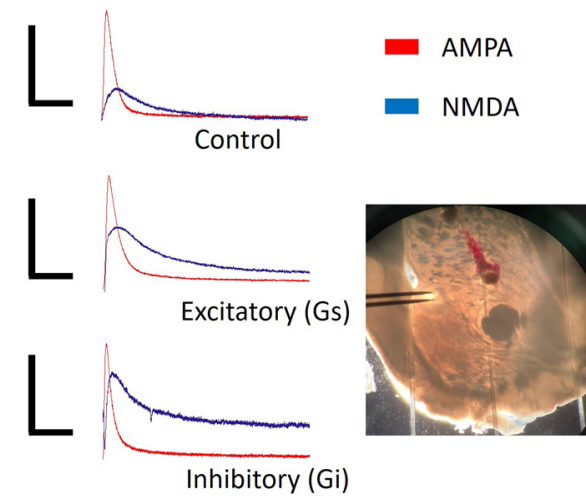

Figure 4. Chemogenetic $\mathrm{Chl}$ inhibition prevented cue-induced nicotine seeking and reduced $\mathrm{A} / \mathrm{N}$ ratio. $\boldsymbol{A}$, The DREADD constructs used in the current study readily express mCherry in neurons (left panel). ChAT labeled cell bodies within the NAcore (middle panel), which co-expressed with all mCherry labeled neurons (right panel). Arrows depict cell bodies. Scale bar: $30 \mu \mathrm{m}$. B, CNO bath application had no effect on control virus expressing Chls (top; black), promoted firing in the excitatory DREADD-expressing Chl (middle; green); and blunted firing in the inhibitory DREADD-expressing Chl (bottom; red). $\boldsymbol{C}$, In control and excitatory DREADD-expressing animals, active lever pressing was increased during cue-induced reinstatement compared with extinction following intra-NAcore CNO treatment. In animals expressing the inhibitory DREADD, CNO inhibition of Chls prevented cue-induced nicotine reinstatement, where active lever pressing during reinstatement was not different from extinction; ${ }^{*} p<0.05$ versus extinction, ${ }^{* *} p<0.01$ versus extinction; n.s., non-significant. Inset numbers represent number of animals. $\boldsymbol{D}, \mathrm{A} / \mathrm{N}$ ratio was reduced in animals with Chl inhibition following reinstatement $[T($ time $)=15]$ relative to control DREADD-expressing animals. The inset numbers within $\boldsymbol{D}$ represent number of animals with the total number of cells recorded from within parentheses. Representative AMPA and NMDA traces for each DREADD type is shown in panel $\boldsymbol{E}$. A picture depicting the NAcore and stimulating electrode placement is also shown. Numbers in bars represent animal number and numbers in parentheses represent the total number of cells. Data points within $\boldsymbol{C}$, $\boldsymbol{D}$ represent individual animal values. Scale bars $(x, y): 50 \mathrm{~ms}, 100 \mathrm{pA} ;{ }^{* *} p<0.01$. 

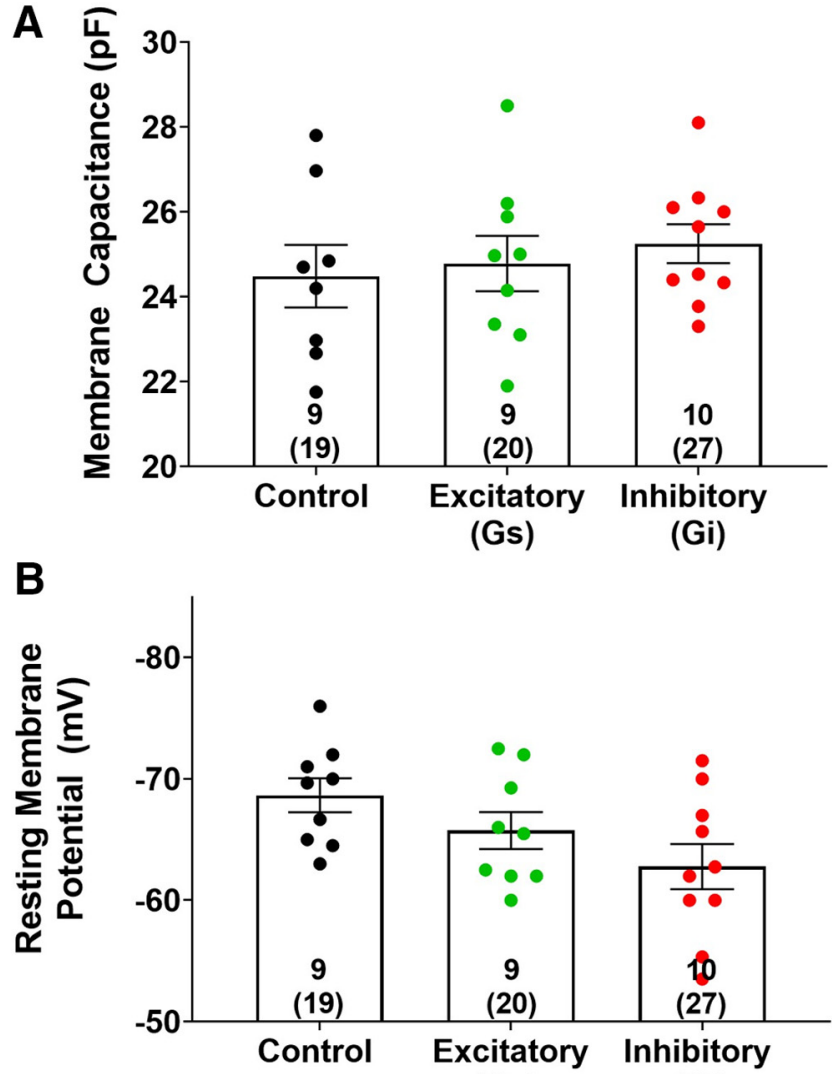

C

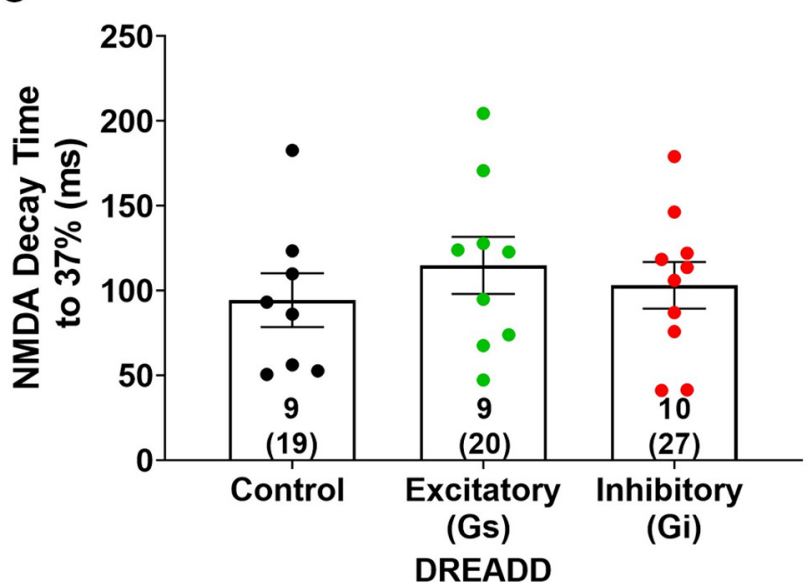

Figure 5. Cellular capacitance, resting membrane potential and NMDA decay time did not differ between DREADD-expressing groups. Cellular capacitance $(\boldsymbol{A})$, resting membrane potential $(\boldsymbol{B})$, and NMDA decay to $37 \%$ of the peak amplitude $(\boldsymbol{C})$ did not differ between control, excitatory, or inhibitory DREADD-expressing animals following cue-induced reinstatement. The inset numbers represent number of animals with the total number of cells recorded from within parentheses. Data points within represent individual animal values.

application of CNO $(10 \mu \mathrm{m})$ did not alter the resting membrane or spiking in control mCherry-expressing Chls, induced firing in excitatory $\mathrm{G}_{\mathrm{s}}$ - DREADD-expressing Chls and inhibited action potential firing in inhibitory $\mathrm{G}_{\mathrm{i}-\mathrm{DREADD}-\mathrm{ex}-}$ pressing Chls (Fig. 4B).

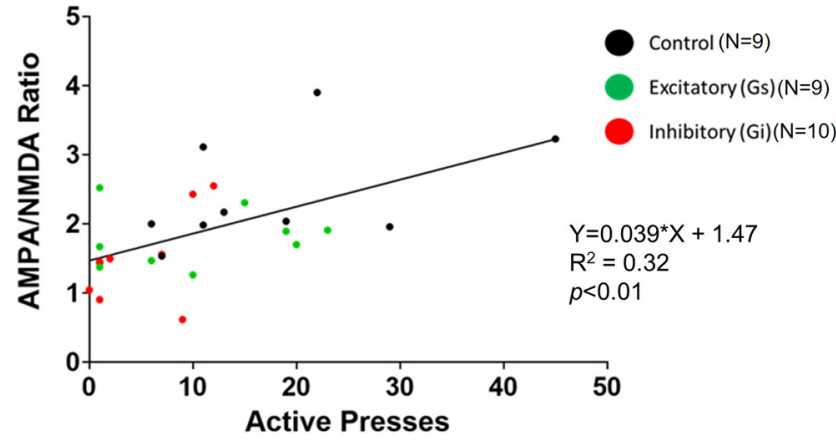

Figure 6. Active lever presses during reinstatement was positively correlated with MSN A/N ratio. The number of active lever presses and $\mathrm{A} / \mathrm{N}$ ratio for each animal is shown. The number of animals plotted for each group is depicted as $N$ within the figure. The $\mathrm{A} / \mathrm{N}$ ratio measured for each cell was averaged across all cells from the same animal (between one and four cells/animal) to obtain the $\mathrm{A} / \mathrm{N}$ for each animal which was then compared with the number of active lever presses during the 15min reinstatement session. Group assignment is depicted as follows: control DREADD-expressing rats are shown as black dots, excitatory DREADD-expressing rats are shown as green, and inhibitory DREADD-expressing rats are shown as red.

\section{Chl inhibition prevents cue-induced nicotine reinstatement}

A two-way ANOVA with treatment (DREADD type) as between- and session (extinction vs reinstatement) as within-subject variables revealed a main effect of treatment $\left(F_{(2,70)}=8.4 ; p<0.01\right.$; Fig. $\left.4 C\right)$ and session $\left(F_{(2,70)}=\right.$ 13.2; $p<0.01$; Fig. $4 C)$. Additionally, an interaction between treatment and session was observed $\left(F_{(2,70)}=6.8\right.$; $p<0.01$; Fig. 4C). Bonferroni post hoc multiple comparisons revealed that control $\mathrm{CNO}$-treated rats increased active lever pressing during cue-induced reinstatement $(T=15)$ relative to the first $15 \mathrm{~min}$ of extinction $\left(t_{(70)}=4.4\right.$; $p<0.01$; Fig. 4C). Additionally, Chl activation with intraNAcore administration of CNO did not prevent cue-induced reinstatement in animals expressing the excitatory DREADD, as the number of active lever presses during the cue-induced reinstatement session $(T=15)$ was higher than that observed during the first 15 min of extinction $\left(t_{(70)}=2.2 ; p<0.05\right.$; Fig. $\left.4 C\right)$. However, inhibition of Chls with CNO prevented cue-induced nicotine reinstatement in inhibitory DREADD-expressing animals, where the number of active lever presses during reinstatement $(T=15)$ was not different from the first $15 \mathrm{~min}$ of extinction $(p>0.05$; Fig. 4C). No differences in inactive lever pressing were observed relative to extinction for any group (data not shown). No sex differences in active lever pressing within groups were observed $(p>0.05)$.

\section{Inhibition of Chls reduces the $\mathrm{A} / \mathrm{N}$ ratio observed in MSNs}

Immediately following a 15-min cue-induced reinstatement session, NAcore MSN recordings were conducted from acute slices derived from animals expressing either control, inhibitory, or excitatory DREADD constructs. An ANOVA revealed a main effect of treatment (DREADD 
type) on $\mathrm{A} / \mathrm{N}$ ratio $\left(F_{(2,25)}=5.7 ; p<0.01\right.$; Fig. $\left.4 D\right)$. Post hoc comparisons revealed that inhibition of $\mathrm{Chl}$ activity resulted in a smaller $\mathrm{A} / \mathrm{N}$ relative to control animals following the 15-min cue-induced reinstatement session $\left(t_{(25)}=3.3\right.$; $p<0.01$; Fig. 4D). However, no differences were observed between rats in which Chls were activated relative to any other group ( $p>0.05$; Fig. $4 D$ ). Additionally, oneway ANOVAs revealed no differences in MSN cellular capacitance $(p>0.05$; Fig. $5 A$ ), resting membrane potential $(p>0.05$; Fig. $5 B)$, or NMDA decay time, as measured by the time to reach $37 \%$ of peak amplitude $(p>0.05$; Fig. $5 C$ ), between control, excitatory or inhibitory DREADDexpressing animals. No sex differences in A/N or NMDA decay within groups were observed $(p>0.05)$.

\section{Active lever pressing during reinstatement is positively correlated with $\mathrm{MSN}$ A/N ratio}

The number of active lever presses and $\mathrm{A} / \mathrm{N}$ were plotted for each animal. The A/N ratio measured for each cell was averaged across all cells from the same animal (between one and four cells/animal) to obtain the $\mathrm{A} / \mathrm{N}$ for each animal. A linear regression analysis revealed a relationship between the number of active lever presses during reinstatement $(T=15)$ and the $\mathrm{A} / \mathrm{N}$ ratio $\left(F_{(1,26)}=12.4\right.$; $p<0.01$; Fig. 6). No sex differences in the slope were observed ( $p>0.05$; data not shown).

\section{Discussion}

The present results show that chemogenetic inhibition of NAcore Chls inhibited cue-induced nicotine-seeking behavior. Additionally, inhibition of Chls reduced $\mathrm{A} / \mathrm{N}$ relative to control animals receiving the same dose of CNO. Further, the magnitude of reinstated nicotine seeking was positively correlated with the $\mathrm{A} / \mathrm{N}$ ratio, where higher active lever pressing was associated with a larger $\mathrm{A} / \mathrm{N}$ ratio, an effect similar to those observed in other studies (Gipson et al., 2013a). Together, these results demonstrate that accumbens Chls exert control over cue-induced nicotine-seeking behavior and MSN synaptic physiology within the NAcore (Marchi et al., 2002).

\section{Interactions between Chls, ACh, nAChRs, and drug- motivated behavior}

The cholinergic system has been heavily implicated in reward-related behaviors. Interestingly, elevated levels of ACh have been observed in the nucleus accumbens following cocaine (Crespo et al., 2006; Yee et al., 2011), remifentanil (Crespo et al., 2006), nicotine (Rada et al., 2001; Mansvelder et al., 2003), and alcohol (Larsson et al., 2005). Further, elevated levels of ACh in the accumbens parallel the reinforcing effects of both intravenous cocaine and remifentanil (Crespo et al., 2006), providing evidence for ACh mediation of drug-motivated behavior. Interestingly, ACh release in the accumbens is also enhanced during contingent versus non-contingent intravenous cocaine administration, suggesting that $A C h$ release may be differentially mediated by volitional versus passive drug exposure (Mark et al., 1999). Chls are also heavily implicated in stimulus-response associations (Aosaki et al., 1994; Suzuki et al., 2001; Joshua et al., 2008; Collins et al., 2019), thus it is not surprising that silencing accumbens Chls prevents cocaine conditioned reward (Witten et al., 2010). Witten and colleagues suggest that acute silencing of accumbens Chls disrupts drug-related learning without affecting conditioning (Witten et al., 2010). Thus, while ACh release is primarily driven by activation of Chls within the accumbens, acute silencing of Chls in this region can disrupt drug-related learning. ACh-releasing Chls have also been shown to exert powerful modulatory control over MSNs and dopaminergic tone within the NAcore (Cachope et al., 2012; Yorgason et al., 2017). Within the current study, we report that inhibition of Chls prevented cue-induced nicotine reinstatement, further suggesting that inhibiting $\mathrm{ACh}$ release within the NAcore may prevent nicotine seeking in part by blunting the stimulus-response association.

nAChRs are densely expressed on Chls as well as glutamatergic and dopaminergic terminals throughout the NAcore (Zhou et al., 2001; Zhang and Sulzer, 2004; Zappettini et al., 2014; Girasole and Nelson, 2015), allowing for extensive control of accumbens circuitry. Further, nAChRs have been heavily implicated in the molecular aspects underlying addiction. For example, nAChRs are upregulated within the accumbens following voluntary ethanol consumption (Larsson et al., 2005). Moreover, $\mathrm{nAChR}$ inhibition also reduces cocaine place preference and cocaine sensitivity (Zachariou et al., 2001) and nAChR antagonism within the VTA attenuates cue-induced cocaine seeking (Nunes et al., 2019). While the role of nAChRs have been explored across multiple drugs of abuse, their involvement in nicotine use and addiction has been most prominently studied. Nicotine directly activates and leads to prolonged desensitization of nAChRs, which contributes to the reinforcing properties of nicotine (Picciotto et al., 1998, 2008; Mansvelder and McGehee, 2002; Mansvelder et al., 2003; Giniatullin et al., 2005). Multiple studies have shown that antagonism of nAChRs reduces active lever pressing in animals self-administering intravenous nicotine (Watkins et al., 1999; Markou and Paterson, 2001; Toll et al., 2012; Liu, 2014), although nAChR modulation specifically within the NAcore in a cue-induced nicotine reinstatement paradigm following self-administration has yet to be studied. Regardless, the findings of these highlighted prior studies support the hypothesis that the NAcore cholinergic system plays a critical role in nicotine addiction.

\section{Interactions between Chls, dopamine release, and MSN synaptic plasticity}

While the main input of MSNs is glutamatergic innervation from the cortex, Chls also receive excitatory cortical inputs. Interestingly, cortical stimulation evokes excitatory responses in Chls before MSNs, as depicted by postsynaptic current latencies and rise times. Specifically, the postsynaptic current latency was slower in MSNs relative to Chls, indicating that Chls are excited by excitatory inputs before MSNs (Fino et al., 2008). With MSNs being downstream targets of Chls, cortical inputs thus have the ability to directly and indirectly modulate the excitability 
and membrane potential of MSNs through direct synaptic connectivity and Chls, respectively. Given these findings, Chls may have the ability to alter MSN synaptic plasticity even before MSNs receiving cortical input. As such, alterations in MSN synaptic plasticity may be because of modifications in the timing and order of the presynaptic and postsynaptic activity at cortico-striatal synapses. In addition to Chls ability to directly alter MSN synaptic excitability, Chls modulate dopamine release within the accumbens by activating nAChRs located on dopaminergic terminals (Nelson et al., 2014). Specifically, synchronous activity of the Chl population can induce DA release by bypassing action potential generation within the dopaminergic soma (Cachope et al., 2012; Threlfell et al., 2012). Not surprisingly, cortical glutamatergic inputs targeting the accumbens can modify dopamine release indirectly through activation of ionotropic glutamate receptors located on Chls (Kosillo et al., 2016). MSNs are particularly susceptible to dopaminergic modulation, where they are distinguished into two major subtypes, classified by their expression of either D1-type or D2-type dopamine receptors. Given their extensive expression of dopamine receptors, dopamine has the ability to extensively modify both the structure and synaptic function of striatal circuits. Current hypotheses suggest that dopaminergic neurons innervating the striatum modify the strength of corticostriatal synapses of MSNs, contributing to action selection behaviors (Yin and Knowlton, 2006; Cohen and Frank, 2009) such as drug seeking.

In the current study, we report that inhibition of Chls reduced the $\mathrm{A} / \mathrm{N}$ ratio of $\mathrm{MSN}$ relative to control, where $\mathrm{Chl}$ activity was unaltered. Given the complexity of this circuitry, it is difficult to pinpoint the exact mechanism driving the observed changes in $\mathrm{A} / \mathrm{N}$ ratio of $\mathrm{MSN}$ in the current study. However, the reduction in Chl activity because of chemogenetic inhibition may prevent pertinent crosstalk between glutamatergic inputs, Chls, dopamine release, and MSNs, resulting in a reduction of $A / N$ ratios in MSNs. Additionally, here we report that activation of Chls reduced the $\mathrm{A} / \mathrm{N}$ ratio, consistent with a recent study showing that $\mathrm{Chl}$ activation during the extinction phase of cocaine CPP reduced the A/N ratio of MSNs, although not relative to controls (Lee et al., 2016). Furthermore, the same study reported a reduction in miniature EPSPs in MSNs as a result of Chl activation, suggesting a reduction in MSN excitatory tone (Lee et al., 2016). However, it may also be possible that chemogenetic activation of Chls did not promote reinstatement beyond control virus-expressing animals in the current study because of $\mathrm{Chl}$ autoregulation, where Chl overexcitation and enhanced ACh release induced by $\mathrm{CNO}$ may lead to activation of muscarinic M4 autoreceptors located on Chls. Activation of these receptors induces membrane hyperpolarization and inhibition of $\mathrm{Chl}$ calcium channels (Girasole and Nelson, 2015), allowing for self-regulation and a blunting of further $\mathrm{ACh}$ release. Because of overexcitation of Chl activity caused by CNO, autoregulatory mechanisms may have prevented Chls from promoting glutamate release and altering MSN synaptic plasticity. However, because these mechanisms were not examined within the current manuscript, future studies testing these mechanisms are warranted.

\section{Limitations of the current study}

While the current study used chemogenetics to explore the effects of Chls on nicotine-seeking and MSN synaptic plasticity, the effects of Chls on other components within the accumbens circuitry including the glutamatergic inputs from the cortex as well as dopamine terminals were not explored. As discussed above, the synaptic connectivity of MSNs has been shown to be altered by Chls, dopamine release, as well as cortical inputs. Thus, to fully characterize the role of Chls in mediating nicotine seeking and accumbens plasticity, additional studies addressing the interactions of Chl-dopamine-MSN interactions as well as MSN subtypes are warranted. These additional studies would further uncover key components contributing to MSN synaptic modifications within the NAcore observed in the current study as well as the regulatory role of the cholinergic system in mediating nicotine-seeking behavior.

Recent studies have demonstrated that ChAT::cre rats display elevated levels of the vesicular acetylcholine transporter protein, as well as altered motor, anxiety and attentional task performance (Mantanona et al., 2019). While it is important to note that these effects could affect the conclusions drawn within the current study, we have recently compared the self-administration characteristics of wild-type Long-Evans rats with ChAT::cre-positive Long-Evans rats and found no differences in total infusions across self-administration sessions or active and inactive lever pressing (LeyrerJackson et al., 2020). Thus, while others have reported behavioral differences of ChAT::cre rats compared with wild type (Mantanona et al., 2019), we believe that the lack of differences in self-administration characteristics demonstrate that these groups do not differ in their nicotine-seeking behaviors. However, future studies would benefit from comparing the MSN synaptic physiology and accumbens circuitry between ChAT::cre and wildtype rats.

In conclusion, we report that $\mathrm{Chl}$ inhibition prevents cue-induced nicotine reinstatement and blunts MSN A/N. These results suggest that the cholinergic system heavily modulates nicotine-seeking behavior and associated glutamate plasticity. Thus, Chls may be an essential modulator of MSN synaptic plasticity, which may play a role in nicotine seeking, that has been previously overlooked. Taken together, the current findings illustrate an additional component of the highly complex neurophysiological underpinnings of nicotine relapse, where Chls mediate control of MSN A/N and thus play a pertinent role in transitioning nicotine craving to seeking.

\section{References}

Alasmari F, Al-Rejaie SS, AISharari SD, Sari Y (2016) Targeting glutamate homeostasis for potential treatment of nicotine dependence. Brain Res Bull 121:1-8. 
Aosaki T, Tsubokawa H, Ishida A, Watanabe K, Graybiel AM, Kimura M (1994) Responses of tonically active neurons in the primate's striatum undergo systematic changes during behavioral sensorimotor conditioning. J Neurosci 14:3969-3984.

Atallah HE, McCool AD, Howe MW, Graybiel AM (2014) Neurons in the ventral striatum exhibit cell-type-specific representations of outcome during learning. Neuron 82:1145-1156.

Brown MT, Bellone C, Mameli M, Labouèbe G, Bocklisch C, Balland B, Dahan L, Luján R, Deisseroth K, Lüscher C (2010) Drug-driven AMPA receptor redistribution mimicked by selective dopamine neuron stimulation. PLoS One 5:e15870.

Brown RW, Kolb B (2001) Nicotine sensitization increases dendritic length and spine density in the nucleus accumbens and cingulate cortex. Brain Res 899:94-100.

Cachope R, Mateo Y, Mathur BN, Irving J, Wang HL, Morales M, Lovinger DM, Cheer JF (2012) Selective activation of cholinergic interneurons enhances accumbal phasic dopamine release: setting the tone for reward processing. Cell Rep 2:33-41.

Cohen MX, Frank MJ (2009) Neurocomputational models of basal ganglia function in learning, memory and choice. Behav Brain Res 199:141-156.

Collins AL, Aitken TJ, Huang IW, Shieh C, Greenfield VY, Monbouquette HG, Ostlund SB, Wassum KM (2019) Nucleus accumbens cholinergic interneurons oppose cue-motivated behavior. Biol Psychiatry 86:388-396.

Crespo JA, Sturm K, Saria A, Zernig G (2006) Activation of muscarinic and nicotinic acetylcholine receptors in the nucleus accumbens core is necessary for the acquisition of drug reinforcement. $J$ Neurosci 26:6004-6010.

Dani JA, Ji D, Zhou FM (2001) Synaptic plasticity and nicotine addiction. Neuron 31:349-352.

Fino E, Deniau JM, Venance L (2008) Cell-specific spike-timing-dependent plasticity in GABAergic and cholinergic interneurons in corticostriatal rat brain slices. J Physiol 586:265-282.

Giniatullin R, Nistri A, Yakel JL (2005) Desensitization of nicotinic ACh receptors: shaping cholinergic signaling. Trends Neurosci 28:371-378.

Gipson CD, Kupchik YM, Shen H, Reissner KJ, Thomas CA, Kalivas PW (2013a) Relapse induced by cues predicting cocaine depends on rapid, transient synaptic potentiation. Neuron 77:867-872.

Gipson CD, Reissner KJ, Kupchik YM, Smith AC, Stankeviciute N, Hensley-Simon ME, Kalivas PW (2013b) Reinstatement of nicotine seeking is mediated by glutamatergic plasticity. Proc Natl Acad Sci USA 110:9124-9129.

Girasole AE, Nelson AB (2015) Probing striatal microcircuitry to understand the functional role of cholinergic interneurons. Mov Disord 30:1306-1318.

Grieder TE, George O, Tan H, George SR, Foll BL, Laviolette SR, van der Kooy D (2012) Phasic D1 and tonic D2 dopamine receptor signaling double dissociate the motivational effects of acute nicotine and chronic nicotine withdrawal. Proc Natl Acad Sci USA 109:3101-3106.

Joshua M, Adler A, Mitelman R, Vaadia E, Bergman H (2008) Midbrain dopaminergic neurons and striatal cholinergic interneurons encode the difference between reward and aversive events at different epochs of probabilistic classical conditioning trials. $J$ Neurosci 28:11673-11684.

Kalivas PW (2009) The glutamate homeostasis hypothesis of addiction. Nat Rev Neurosci 10:561-572.

Kljakic O, Janickova H, Prado VF, Prado MAM (2017) Cholinergic/ glutamatergic co-transmission in striatal cholinergic interneurons: new mechanisms regulating striatal computation. J Neurochem 142 [Suppl 2]:90-102.

Kosillo P, Zhang YF, Threlfell S, Cragg SJ (2016) Cortical control of striatal dopamine transmission via striatal cholinergic interneurons. Cereb Cortex 26:4160-4169.

Larsson A, Edström L, Svensson L, Söderpalm B, Engel JA (2005) Voluntary ethanol intake increases extracellular acetylcholine levels in the ventral tegmental area in the rat. Alcohol Alcohol 40:349358.
Lattal KM, Lattal KA (2012) Facets of Pavlovian and operant extinction. Behav Processes 90:1-8.

Lee J, Finkelstein J, Choi JY, Witten IB (2016) Linking cholinergic interneurons, synaptic plasticity, and behavior during the extinction of a cocaine-context association. Neuron 90:1071-1085.

Leyrer-Jackson JM, Overby PF, Bull A, Marusich JA, Gipson CD (2020) Strain and sex matters: differences in nicotine self-administration between outbred and recombinase-driver transgenic rat lines. Exp Clin Psychopharmacol, in press.

Liu X (2014) Effects of blockade of $\alpha 4 \beta 2$ and $\alpha 7$ nicotinic acetylcholine receptors on cue-induced reinstatement of nicotine-seeking behaviour in rats. Int J Neuropsychopharmacol 17:105-116.

Mahler SV, Aston-Jones G (2018) CNO evil? Considerations for the use of DREADDs in behavioral neuroscience. Neuropsychopharmacology 43:934-936.

Mansvelder HD, McGehee DS (2000) Long-term potentiation of excitatory inputs to brain reward areas by nicotine. Neuron 27:349357.

Mansvelder HD, McGehee DS (2002) Cellular and synaptic mechanisms of nicotine addiction. J Neurobiol 53:606-617.

Mansvelder HD, De Rover M, McGehee DS, Brussaard AB (2003) Cholinergic modulation of dopaminergic reward areas: upstream and downstream targets of nicotine addiction. Eur $\mathrm{J}$ Pharmacol 480:117-123.

Mansvelder HD, Mertz M, Role LW (2009) Nicotinic modulation of synaptic transmission and plasticity in cortico-limbic circuits. Semin Cell Dev Biol 20:432-440.

Mantanona CP, Alsiö J, Elson JL, Fisher BM, Dalley JW, Bussey T, Pienaar IS (2019) Altered motor, anxiety-related and attentional task performance at baseline associate with multiple gene copies of the vesicular acetylcholine transporter and related protein overexpression in ChAT::Cre+ rats. Brain Struct Funct 224:30953116.

Marchi M, Risso F, Viola C, Cavazzani P, Raiteri M (2002) Direct evidence that release-stimulating alpha $7^{*}$ nicotinic cholinergic receptors are localized on human and rat brain glutamatergic axon terminals. J Neurochem 80:1071-1078.

Mark GP, Hajnal A, Kinney AE, Keys AS (1999) Self-administration of cocaine increases the release of acetylcholine to a greater extent than response-independent cocaine in the nucleus accumbens of rats. Psychopharmacology (Berl) 143:47-53.

Markou A, Paterson NE (2001) The nicotinic antagonist methyllycaconitine has differential effects on nicotine self-administration and nicotine withdrawal in the rat. Nicotine Tob Res 3:361-373.

Namba MD, Kupchik YM, Spencer SM, Garcia-Keller C, Goenaga JG, Powell GL, Vicino IA, Hogue IB, Gipson CD (2020) Accumbens neuroimmune signaling and dysregulation of astrocytic glutamate transport underlie conditioned nicotine-seeking behavior. Addict Biol 25:e12797.

Nelson AB, Hammack N, Yang CF, Shah NM, Seal RP, Kreitzer AC (2014) Striatal cholinergic interneurons drive GABA release from dopamine terminals. Neuron 82:63-70.

Nunes EJ, Bitner L, Hughley SM, Small KM, Walton SN, Rupprecht LE, Addy NA (2019) Cholinergic receptor blockade in the VTA attenuates cue-induced cocaine-seeking and reverses the anxiogenic effects of forced abstinence. Neuroscience 413:252-263.

Oldenburg IA, Ding JB (2011) Cholinergic modulation of synaptic integration and dendritic excitability in the striatum. Curr Opin Neurobiol 21:425-432.

Overby PF, Daniels CW, Del Franco A, Goenaga J, Powell GL, Gipson CD, Sanabria F (2018) Effects of nicotine self-administration on incentive salience in male Sprague Dawley rats. Psychopharmacology (Berl) 235:1121-1130.

Paxinos G, Franklin KBJ (2001) The mouse brain in stereotaxic coordinates. Orlando: Academic Press.

Picciotto MR, Zoli M, Rimondini R, Léna C, Marubio LM, Pich EM, Fuxe K, Changeux JP (1998) Acetylcholine receptors containing the beta2 subunit are involved in the reinforcing properties of nicotine. Nature 391:173-177. 
Picciotto MR, Addy NA, Mineur YS, Brunzell DH (2008) It is not "either/or": activation and desensitization of nicotinic acetylcholine receptors both contribute to behaviors related to nicotine addiction and mood. Prog Neurobiol 84:329-342.

Pistillo F, Clementi F, Zoli M, Gotti C (2015) Nicotinic, glutamatergic and dopaminergic synaptic transmission and plasticity in the mesocorticolimbic system: focus on nicotine effects. Prog Neurobiol 124:1-27.

Prochaska JJ, Benowitz NL (2016) The past, present, and future of nicotine addiction therapy. Annu Rev Med 67:467-486.

Quintero GC (2013) Role of nucleus accumbens glutamatergic plasticity in drug addiction. Neuropsychiatr Dis Treat 9:1499-1512.

Rada P, Jensen K, Hoebel BG (2001) Effects of nicotine and mecamylamine-induced withdrawal on extracellular dopamine and acetylcholine in the rat nucleus accumbens. Psychopharmacology (Berl) 157:105-110.

Rescorla RA, Skucy JC (1969) Effect of response independent reinforcers during extinction. J Comp Physiol Psychol 67:381-389.

Scofield MD, Heinsbroek JA, Gipson CD, Kupchik YM, Spencer S, Smith AC, Roberts-Wolfe D, Kalivas PW (2016) The nucleus accumbens: mechanisms of addiction across drug classes reflect the importance of glutamate homeostasis. Pharmacol Rev 68:816-871.

Stefanik MT, Kupchik YM, Kalivas PW (2016) Optogenetic inhibition of cortical afferents in the nucleus accumbens simultaneously prevents cue-induced transient synaptic potentiation and cocaineseeking behavior. Brain Struct Funct 221:1681-1689.

Suzuki T, Miura M, Nishimura K, Aosaki T (2001) Dopamine-dependent synaptic plasticity in the striatal cholinergic interneurons. J Neurosci 21:6492-6501.

Tepper JM, Bolam JP (2004) Functional diversity and specificity of neostriatal interneurons. Curr Opin Neurobiol 14:685-692.

Threlfell S, Lalic T, Platt NJ, Jennings KA, Deisseroth K, Cragg SJ (2012) Striatal dopamine release is triggered by synchronized activity in cholinergic interneurons. Neuron 75:58-64.

Toll L, Zaveri NT, Polgar WE, Jiang F, Khroyan TV, Zhou W, Xie XS, Stauber GB, Costello MR, Leslie FM (2012) AT-1001: a high affinity and selective $\alpha 3 \beta 4$ nicotinic acetylcholine receptor antagonist blocks nicotine self-administration in rats. Neuropsychopharmacology 37:1367-1376.

Tomek SE, Stegmann GM, Leyrer-Jackson JM, Piña J, Olive MF (2020) Restoration of prosocial behavior in rats after heroin selfadministration via chemogenetic activation of the anterior insular cortex. Soc Neurosci 15:408-419.

Watkins SS, Epping-Jordan MP, Koob GF, Markou A (1999) Blockade of nicotine self-administration with nicotinic antagonists in rats. Pharmacol Biochem Behav 62:743-751.

Witten IB, Lin SC, Brodsky M, Prakash R, Diester I, Anikeeva P, Gradinaru V, Ramakrishnan C, Deisseroth K (2010) Cholinergic interneurons control local circuit activity and cocaine conditioning. Science 330:1677-1681.

Yee J, Famous KR, Hopkins TJ, McMullen MC, Pierce RC, Schmidt HD (2011) Muscarinic acetylcholine receptors in the nucleus accumbens core and shell contribute to cocaine priming-induced reinstatement of drug seeking. Eur J Pharmacol 650:596-604.

Yin HH, Knowlton BJ (2006) The role of the basal ganglia in habit formation. Nat Rev Neurosci 7:464-476.

Yorgason JT, Zeppenfeld DM, Williams JT (2017) Cholinergic interneurons underlie spontaneous dopamine release in nucleus accumbens. J Neurosci 37:2086-2096.

Zachariou V, Caldarone BJ, Weathers-Lowin A, George TP, Elsworth JD, Roth RH, Changeux JP, Picciotto MR (2001) Nicotine receptor inactivation decreases sensitivity to cocaine. Neuropsychopharmacology 24:576-589.

Zappettini S, Grilli M, Olivero G, Chen J, Padolecchia C, Pittaluga A, Tomé AR, Cunha RA, Marchi M (2014) Nicotinic $\alpha 7$ receptor activation selectively potentiates the function of NMDA receptors in glutamatergic terminals of the nucleus accumbens. Front Cell Neurosci 8:332.

Zhang H, Sulzer D (2004) Frequency-dependent modulation of dopamine release by nicotine. Nat Neurosci 7:581-582.

Zhou FMCJ, Wilson JA Dani (2002) Cholinergic interneuron characteristics and nicotinic properties in the striatum. J Neurobiol 53:590-605.

Zhou FM, Liang Y, Dani JA (2001) Endogenous nicotinic cholinergic activity regulates dopamine release in the striatum. Nat Neurosci $4: 1224-1229$ 